\title{
Developing reliable reaction gamma-ray data
}

\author{
Paraskevi Dimitriou ${ }^{1, *}, T$. Belgya ${ }^{2}, Y-S$. Cho $^{3}, D$. Filipescu $^{4}, R$. Firestone ${ }^{5}, S$. Goriely ${ }^{6}$, \\ $N$. Iwamoto ${ }^{7}, T$. Kawano ${ }^{8}, J$. Kopecky ${ }^{9}, M . \mathrm{Krticka}^{10}, V$. Plujko ${ }^{11}, S$. Siem ${ }^{12}$, \\ $R$. Schwengner ${ }^{13}, H$. Utsunomiya ${ }^{14}, V$. Vlarlamov ${ }^{15}, M$. Wiedeking ${ }^{16}, R . \mathrm{Xu}^{17}$ \\ ${ }^{1}$ International Atomic Energy Agency, Wagramerstrasse 5, A-1400 Vienna, Austria \\ ${ }^{2}$ Centre for Energy Research, Hungarian Academy of Sciences, Budapest, Hungary \\ ${ }^{3}$ Nuclear Data Center, Korea Atomic Energy Research Institute, Daejeon, Rep. of Korea \\ ${ }^{4}$ Horia Hulubei National Institute, Bucuresti-Magurele, Romania \\ ${ }^{5}$ University of California at Berkeley, Berkeley, California 94720, USA \\ ${ }^{6}$ Universite Libre de Bruxelles, CP-226, Campus de la Plaine, 1050 Brussels, Belgium \\ ${ }^{7}$ Japan Atomic Energy Agency (JAEA), Tokai-mura, Ibaraki 319-1195, Japan \\ ${ }^{8}$ Los Alamos National Laboratory, New Mexico, 87454, USA \\ ${ }^{9}$ JUKO Research, Kalmanstraat 4, Alkmaar 1817, The Netherlands \\ ${ }^{10}$ Charles University in Prague, 18000 Prague 8, Czech Republic \\ ${ }^{11}$ Taras Shevchenko National University, Kiev 01601, Ukraine \\ ${ }^{12}$ University of Oslo, PO Box 1048, Oslo 0316, Norway \\ ${ }^{13}$ Helmholtz Zentrum Dresden-Rossendorf (HZDR), Dresden, Germany \\ ${ }^{14}$ Konan University, 8-9-1 Okamoto, Higashinada, Japan \\ ${ }^{15}$ Lomonosov Moscow State University, 119991 Moscow, Russian Federation \\ ${ }^{16}$ iThemba LABS, PO Box 2600, Pretoria Gauteng 0001, South Africa \\ ${ }^{17}$ China Institute of Atomic Energy, PO Box 275(41), Beijing, China
}

\begin{abstract}
We report on efforts to develop reliable photonuclear cross section and photon strength function data by measuring, compiling, assessing, evaluating the available data, and producing tables of Giant Dipole Resonance parameters and global models for use in basic sciences and applications.
\end{abstract}

\section{Motivation}

Gamma-ray data from nuclear reactions are important for a large range of applications, as well as for basic sciences. In particular, gamma-ray data to extract photon strength functions and photonuclear cross sections are necessary for energy, safety and medical applications as well as for nuclear physics and astrophysics.

There is a wealth of reaction gamma-ray data related to photonuclear reactions and photon strength functions published in recent years that needs to be compiled and evaluated, and made available to researchers worldwide.

In this work we report on the international effort under an IAEA Coordinated Research Project (CRP) [1] to compile all the reaction gamma-ray data and assess their reliability on the basis of measurement techniques and systematic comparisons with theoretical models.

* Corresponding author: p.dimitriou@iaea.org 


\section{Photonuclear cross sections}

The IAEA Photonuclear data library (http://www-nds.iaea.org/photonuclear) includes photoabsorption cross sections, total and partial photo-neutron reaction cross sections, and neutron emission energy spectra for 164 isotopes. Since its release in 1999, new data have been measured, improved evaluation techniques and theories have been developed and new measurement techniques aiming at resolving the existing discrepancies have emerged.

The current IAEA CRP is updating the photonuclear data library by revising the existing evaluations and adding 37 new evaluations of isotopes that were not included before. Additionally, photo-production of isotopes of interest to medical applications are also being considered, such as isotopes produced by $\gamma+{ }^{39,41} \mathrm{~K},{ }^{186} \mathrm{Re}$ produced via ${ }^{187} \operatorname{Re}(\gamma, \mathrm{n}){ }^{186} \mathrm{Re}$ for which data exist.

Existing evaluation approaches and models implemented in the different reaction codes are being assessed and their impact on the evaluations is explored. A systematic comparison of the correction functions $F_{i}$-often used as criteria for reliability of $(\gamma, \mathrm{xn})$ data [2]-produced by six nuclear reaction codes used in the evaluations is shown in Fig. 1. The figure clearly indicates that this approach depends on both the reaction models and input parameters used in the calculations, and that the differences between them vary with energy. The $F_{i}$ evaluation approach should therefore be applied by considering these modeldependent uncertainties.
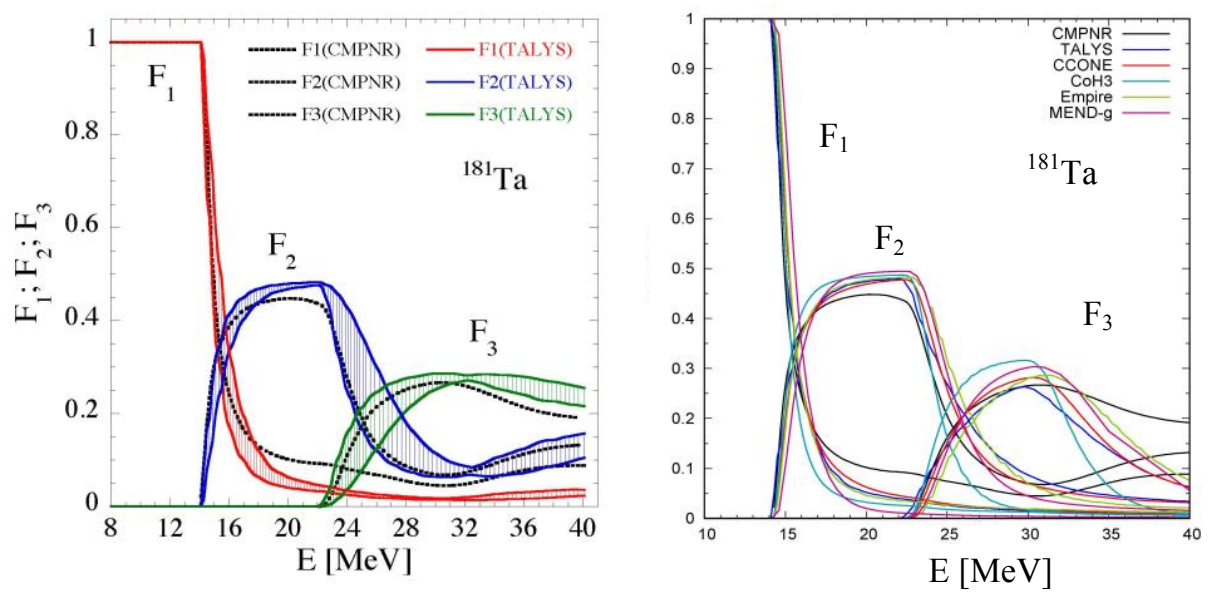

Fig. 1. $F_{1}, F_{2}, F_{3}$ correction functions for partial photoneutron cross section $(\gamma$, in $)$ on ${ }^{181}$ Ta. Left panel: shaded areas correspond to TALYS calculations using different nuclear level densities and photon strength functions. Right panel: coloured curves correspond to results from CMPNR, TALYS, CCONE, CoH3, Empire 3.2, MEND-g codes. For more details see [1].

In parallel with the evaluations, the new multiplicity counting technique used at the NewSUBARU facility with the aim of resolving the long-standing discrepancies between the existing photoneutron multiplicity data is also producing interesting results [3].

Finally, GDR parameters have been extracted by fitting Lorentzian-type curves to a revised database of experimental photoabsorption and photoneutron cross-section data published up to June 2017. The new compilation is an extension and improvement of earlier compilations and also includes recommendations [4]. 


\section{Photon Strength Functions}

Photon strength functions extracted from different measurements have been compiled and assessed. The compilation includes data in the GDR region and in the range near and below the neutron separation energy, including average resonance capture data [5]. The data are being assessed to ensure compatibility near the neutron thresholds, as well as to resolve discrepancies observed between different types of measurements and extraction methods, such as $(\gamma, \gamma)$ and data obtained with the Oslo method. Global models (Lorentzian-type SLO/SMLO [4], QRPA [6]) have also been developed in the entire energy range. Figure 2 shows such an example where photon strength data from different sources are compared. All these data will be made available through a user-friendly web interface provided by the IAEA.

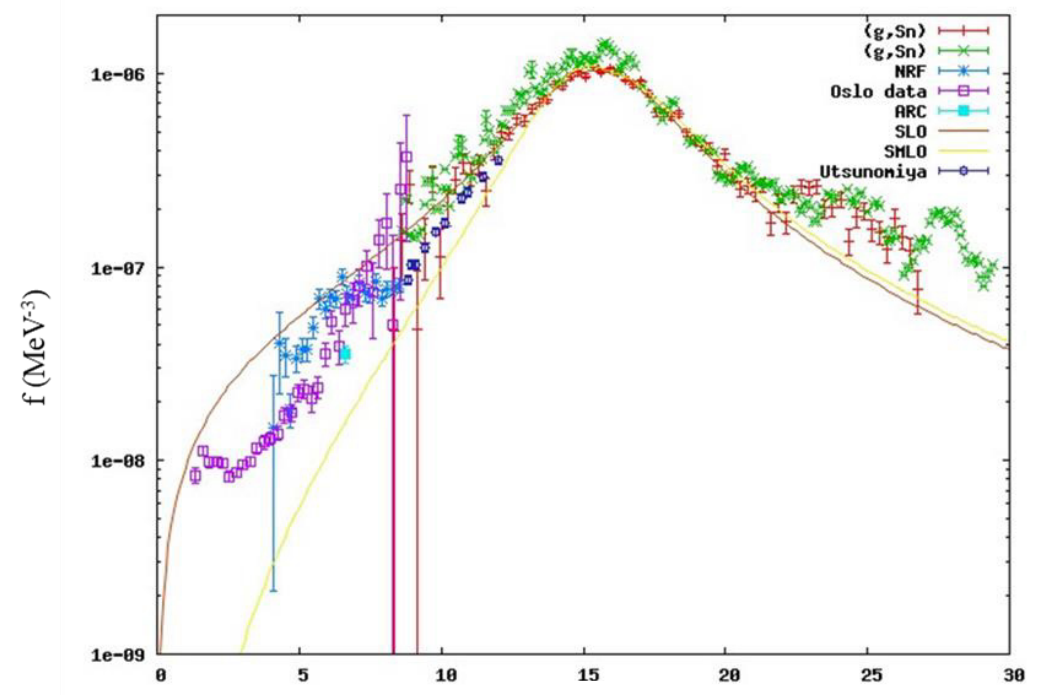

Fig. 2. Photon strength functions $\left(\mathrm{MeV}^{-3}\right)$ for ${ }^{98} \mathrm{Mo}$ from $(\gamma, \mathrm{abs})$, average resonance capture data, $(\gamma, \mathrm{n})$, $(\gamma, \gamma)$ and $\left({ }^{3} \mathrm{He},{ }^{3} \mathrm{He}\right)$ reactions compared with empirical SLO/SMLO functions (see [1] and references therein).

\section{References}

1. S. Goriely, M. Wiedeking and P. Dimitriou, Summary Report of the 2nd RCM of the CRP on Updating the IAEA Photonuclear Data Library and Generating a Reference Database for Photon Strength Functions, IAEA Report INDC(NDS)-0745, Vienna 2017; http://www-nds.iaea.org/publications/indc/indc-nds-0745.pdf

2. V.V. Varlamov, B.S. Ishkhanov, INDC(CCP)-433, Vienna 2002; http://www-nds.iaea.org/publications/indc-ccp-0433.pdf

3. I. Gheorghe et al., Phys. Rev. C 96, 044604 (2017).

4. V.A. Plujko, O.M. Gorbachenko, R. Capote, P. Dimitriou, ADNDT, in press.

5. J. Kopecky, Atlas of Average Resonance Capture Data, INDC(NDS)-0738, Vienna 2017; http://www-nds.iaea.org/pubications/indc/indc-nds-0738.pdf

6. S. Goriely, S. Hilaire, S. Péru, M. Martini, et al., Phys. Rev. C 94, 044306 (2016). 